\title{
RemoTouch: \\ A System For Remote Touch Experience
}

\author{
Domenico Prattichizzo $^{1,2}$ Francesco Chinello $^{1}$ Claudio Pacchierotti ${ }^{1}$ Kouta Minamizawa $^{3}$ \\ ${ }^{1}$ Dipartimento di Ingegneria dell'Informazione, Università di Siena, Italia \\ ${ }^{2}$ Istituto Italiano di Tecnologia, Genova Italia \\ ${ }^{3}$ Tachi Lab, University of Keio, Japan
}

\begin{abstract}
This paper presents some preliminary results on RemoTouch, a system allowing to perform experiences of remote touch. The system consists of an avatar equipped with an instrumented glove and a user wearing tactile displays allowing to feel the remote tactile interaction. The main features of RemoTouch are that it is a wearable system and that a human avatar is used to collect remote tactile interaction data. New paradigms of tactile communication can be designed around the RemoTouch system. Two simple experiences are reported to show the potential of the proposed remote touch architecture.
\end{abstract}

\section{INTRODUCTION}

Humans have always attempted to extend their perception abilities to enlarge the workspace of the human body. Consider, for instance, the role played by mobile phones for audio modality. If one thinks about technology for recording and playback of audio and video, portable devices come to mind. It isn't so for touch modality. Technology for touching remote objects has typically been used in robotics for teleoperation and the devices used are very far from being portable. In telepresence, a robot is used as a slave in the remote scenario and a haptic interface or an exoskeleton feeds back contact forces letting the user perceive the remote environment [1]. Current technology for telepresence is very advanced but it is not portable and is not low energy consumption thus compromising the possibility to be used in everyday life. in Fig. 1 an example of telepresence system developed at DLR is reported [2].

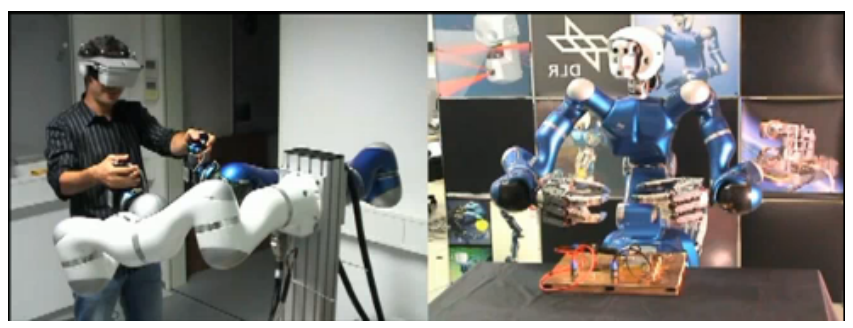

Fig. 1. Example of a teleperation system where the user teleoperates by the remote robot. Courtesy of Institute of Robotics and Mechatronics German Aerospace Center (DLR),

A different approach to remote tactile perception is presented in this work. It involves replacing the slave robot with

e-mail: prattichizzo,chinello,pacchierotti@dii.unisi.it,kouta@tachilab.org a human operator and substituting the exoskeleton, or other haptic interfaces, with a simple and wearable tactile device. In the RemoTouch project the device in charge of recording tactile perception is not a robot but a human avatar. It is able to collect tactile, audio and video signals to be fed back to the remote user. This is what we refer to as "remote perception" in this paper.

Of course, the main difference with teleoperation is that the human avatar cannot be controlled as a robot but this has nothing to do with RemoTouch which only deals with the perception of the remote environment through human avatars.

\section{THE REMoTOUCH IDEA}

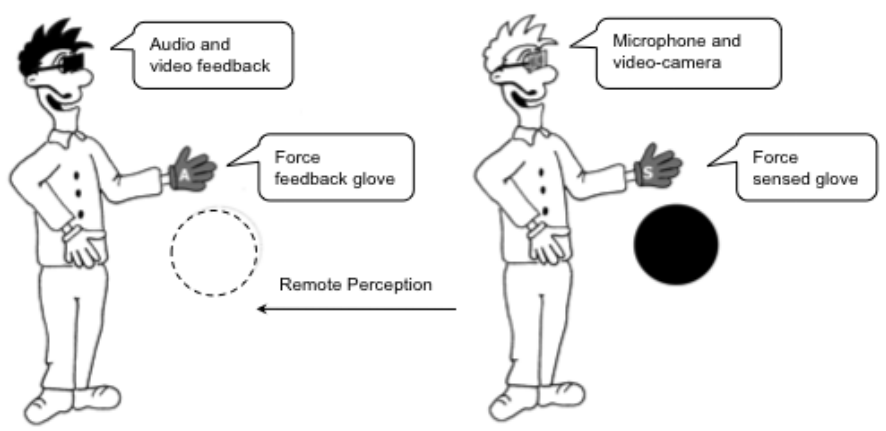

Fig. 2. The functional scheme of the RemoTouch project. A remote human avatar collects data to be fed back to the user.

The idea of RemoTouch is pictorially described in Fig. 2: the user (on the left) perceives the force feedback recorded by the human avatar (on the right). The human avatar wears a glove equipped with force sensors, one per finger as in Fig. 3. The measured contact force at the remote interaction is fed back to the user through simple and wearable tactile displays, one per finger, as in Fig. 3. The tactile display is similar to those developed in [3] and has been adapted to the idea of remote touch here presented. The tactile display consists of two motors and a belt able to deform the fingertip according to the contact force measured by the remote instrumented glove. RemoTouch is a project which involves telepresence and communication between two users 


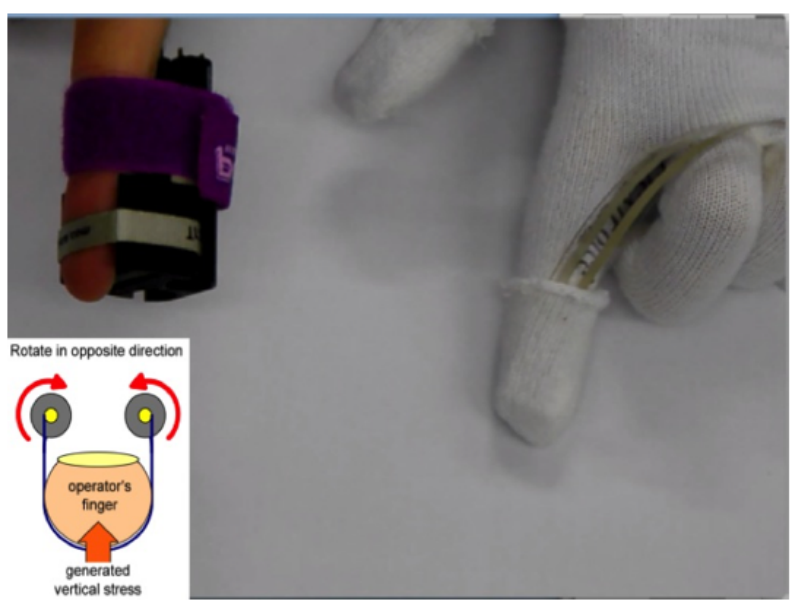

Fig. 3. The glove instrumented with force sensors on the right and the tactile display (with the functional scheme) on the left.

without using a robotic avatar. So the target is to be able to transfer the contact signal in the same way the voice and the video image are sent to the remote user. Note that the force feedback is tactile only and the kinesthetic feedback is missed. Recently, results have been presented to study to what extent the kinesthetic feedback can be substituted with tactile feedback only [4]. Practical experiments have shown that this lack of feedback in force is well compensated by the presence of other modalities like video and audio feedback, which are extensively used in our experiments. A very important feature of RemoTouch is that the involved technology is simple, wearable, low energy consumption and not expensive. Of course this is a direct consequence of having chosen a human avatar instead of a remote robot which is not a secondary point but the main point of our project.

\section{The REMoTouCH SYSTEM}

In this section the hardware and software parts of the system are described.

\section{A. Tactile feedback}

The device in charge of recording tactile perception is not a robot, as in teleoperation, but a human avatar. The human avatar wears a glove equipped with a force sensor. The piezoresistive force sensor by FlexiForce (model A201) has been chosen to equip each finger of the human avatar as in Fig. 3. This force sensor is easily wearable due to its flexible structure and has been integrated with a glove to measure the deformation of the human avatar's fingertip during the contact interaction. A standard protocol based on USB has been used for communication with the computational unit and to provide the power supply.

Regarding the user, the force feedback recorded by the human avatar is presented to the user using a simple and wearable tactile display as shown in Fig. 3. It consists of two motors and a belt able to deform the fingertip according to the contact force measured by the remote instrumented glove. When motors spin in opposite directions the belt applies a perpendicular force on the user's fingertip. Note that this tactile display can also render tangential forces but this is not used in RemoTouch since the force sensors on the human hand measure only normal forces. The tangential forces will be implemented on the next version of the system. For this reason two motors, instead of one, are used to generate the normal force. The motors are current controlled and the torque command is sent by an embedded $\mathrm{C}++$ library. The force feedback is local to the fingerpad and the kinesthetic feedback is missed. [4]. The actuators are a couple of DC motors of $5 \mathrm{~V}$ and $500 \mathrm{~mA}$ maximum current. The maximum torque is about $5.80 \mathrm{~N} \cdot \mathrm{cm}$ so the maximum force applied by the belt on the pad is about $3 \mathrm{~N}$. As far as calibration is concerned, the same force sensor used for the remote instrumented glove has been also used to calibrate the tactile display. The force sensor was fixed between the belt and the fingerpad, as if the same user was using both the instrumented glove and the tactile display at the same time. The relationship between the current to the two motors of the tactile display and the resulting normal force applied at the fingerpad and measured by the FlexiForce sensor is approximately linear as shows in Fig. 4. The relationship used to map the normal forces to motor currents is

$$
c=0.720 f+0.1551
$$

where $f$, in $\mathrm{N}$, is the normal force measured at the remote interaction point with the force sensor fixed to the glove of the remote avatar and $c$ is the current, in $\mathrm{dA}$, to be sent to the motors of the tactile display to render the remote measured normal force at the contact.

\section{B. Visual feedback}

The remote user, who wears the tactile display, enhances his perception using head mounted video-glasses displaying what the head-mounted camera on the human avatar is

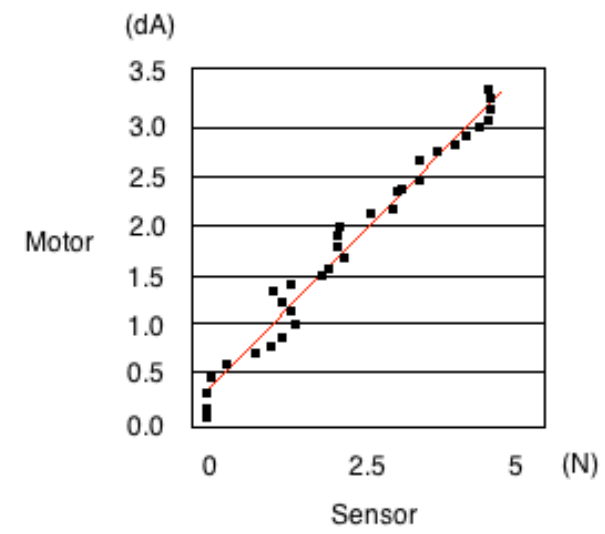

Fig. 4. Tactile display charateristic. Motor current (dA) and normal force (N) generated by the belt on the fingerpad. 
capturing. The video signal is acquired by the human avatar camera and transmitted to the user's head mounted display. Particular attention has been paid to get coherence between the point of view of the head mounted camera and the display.

The visual feedback is a relevant part of the remote touch experience. The experience perceived by the user is justified by a perceptive illusion similar to the one described for the rubber hand in [5]. The main idea is to let the operator perceive the remote objects through the human avatar. Using only the tactile modality, there is not enough information about the remote context. Experiments on brain cognition show that the human brain needs some knowledge about the remote environment [6]. In particular the human avatar needs to share what he's seeing with the remote user using the same point of view of the 3D scene. The RemoTouch vision system consists of a camera, fixed on the head of the human avatar, and a head mounted display worn by the user during the experience. Although the 3D display is an immersive experience for vision system, RemoTouch doesn't use a 3D vision system. This lack is due to the consistent cost of the 3D vision devices and the consequent increase in hardware complexity and portability reduction. The camera position and the multimedia glasses are crucial, they need to be adapted to each single user since, as discussed in [6], the visual information from the first person perspective plays an important role in establishing the location of the perceived body relative to environmental landmarks and in defining the origin of the body centered reference frame.

\section{TWO EXPERIENCES OF REMOTE TOUCH}

Two experiences of remote touch are presented. One of the aims of the RemoTouch project is to improve the quality of communication. Touch is important to communicate feelings and emotions. The aim of the experiences was to test the tactile communication and a possible application where touch could be useful. For these two reasons a family contest and a music training activity were selected. The first experience shows RemoTouch performing the task of touching a baby. Think of a family where the mother with her child are at home and the father is far away. The mother, in this case, wears the instrumented glove to record and transmit the interaction forces while touching her child. The mother also uses a head mounted camera to feed back visual data to the remote husband. The father plays the role of the user and, through the tactile display and the head mounted display, perceives the tactile and visual experience recorded by his wife while touching their child (Fig. 5). Preliminary tests show that the realism of this remote experience largely improve with the tactile feedback.

The context chosen for the remote experience is very important: having a tactile interaction with their own child involves intense emotions thus increasing the sense of presence even if important components like the kinesthetic feedback are missed in the communication.

The second experience deals with playing and listening to music. This is another very involving experience in everyday

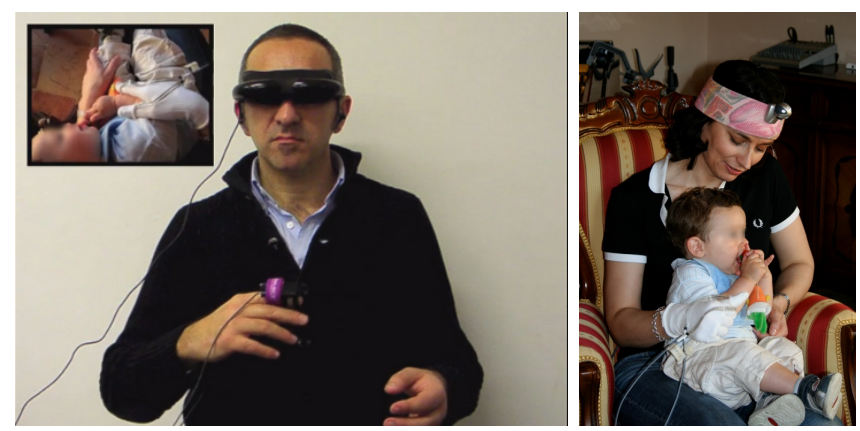

Fig. 5. An experience of remote touch in a context where the mother remotely touches the child and transmits the tactile interaction to the father who is able to perceive the remote touch experience.
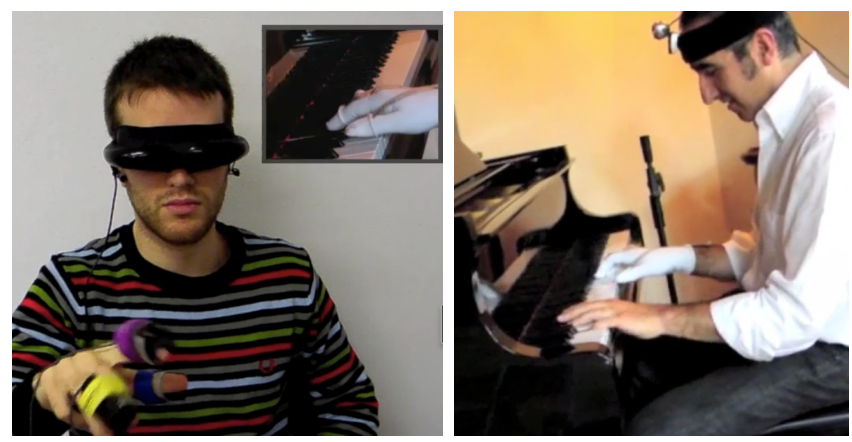

Fig. 6. The second experience of remote touch where a remote piano player transmits the tactile interaction to another player who is able to perceive the remote touch experience.

life. In the experiment, the human avatar plays the piano and records the tactile experience, with the instrumented glove, to be played back to the remote user wearing the tactile displays (Fig. 6). For audio feedback, a microphone is used to capture the audio signal and in-ear headphones have been used for play back.

To perform these experiences a TCP/IP LAN connection has been used. TCP protocol is optimized for accurate delivery rather than timely delivery, and therefore, TCP sometimes incurs in delays while waiting for out-of-order messages or retransmissions of lost messages. Several techniques were used to synchronize the video flow and the tactile feedback recorded by the instrumented glove. The current implementation of RemoTouch uses synchronising signals but we plan to add the support, in the next future, for the Real-time Transport Protocol (RTP) running over the User Datagram Protocol (UDP) that is the now-a-days used protocol for real-time applications such as Voice Over IP.

The video of the two experiences can be found at http://remotouch.dii.unisi.it.

\section{Discussion}

Transmitting tactile information can be used to enhance communication between humans. In particular we can communicate different emotions which cannot be easily transmitted through commonly used communication modalities like audio and video. When RemoTouch is used as a tactile 
communication system, the data-flow consists of recording tactile interaction forces with the instrumented glove to be transmitted to the tactile display along with video and audio signals.

Another very interesting scenario for RemoTouch is to use this system not as a real-time communication system but as a system to record the many visual-tactile experiences to be played back not in real-time but at a different time. These experiences can be shared with other people as it usually happens for images or music in social networks.

A further interesting aspect is to build a database of tactile experiences recorded through RemoTouch. The challenge here is to store tactile data to get information retrieval using simple search engines. We are planning to develop a tactile database where users can submit their recorded experiences using tagging techniques as for audio and video files, e.g. the ID3 tag system for mp3 files.

\section{CONCLUSIONS}

In this paper we presented RemoTouch, a system to perform remote touch experiences. Two example have been presented dealing with particularly emotional contexts.

An important feature of RemoTouch is that the involved technology is low cost and with low energy consumption. Of course this is a consequence of choosing a human avatar which is not a secondary point, but the main point of our project. This work is still in its infancy and we believe that many other applications can be found for RemoTouch. Consider rehabilitation where RemoTouch can be used to easily control the force distribution among fingers in hand grasping tasks for rehabilitation. Substituting the robotic avatar with the human avatar means lower costs, a simplification of the system and a more simple approach for the user. On the other hand, the kinesthetic feedback is missed, so a vision system is used for any position feedback of the human avatar. The weak point of the proposed remote touch architecture is that the performance, especially in terms of realism, of the overall system has been sacrificed in exchange of the portability and wearability of the devices.

We believe that new interaction paradigms based on tactile communication can be developed around RemoTouch for applications in both telepresence and teleoperation. Regarding teleoperation, note that RemoTouch deals only with the remote perception while the action needed to control the remote avatar is an open and very interesting issue and will be the object of future investigations.

\section{ACKNOWLEDGMENTS}

The RemoTouch idea has been selected by the Ministero Italiano dell'Innovazione as one of the Italian innovations within the "Italia degli Innovatori" initiative, organized by the Italian Government for the Shangai 2010 Expo. The authors wish to thank the student Leonardo Meli for his help in realizing the experiments.

This work is supported by the European Commission under CP grant no. 248587, "THE Hand Embodied", within the FP7-ICT-2009-4-2-1 program "Cognitive Systems and Robotics"and CP grant no. 231143, "Experiment HANDS.DVI" within the program "Echord".

\section{REFERENCES}

[1] M. Bergamasco, B. Allotta, L. Bosio, L. Ferretti, G. Parrini, G.M. Prisco, F. Salsedo, and G. Sartini. An arm exoskeleton system for teleoperation and virtual environments applications. In Robotics and Automation, 1994. Proceedings., 1994 IEEE International Conference on, pages $1449-1454$ vol.2, may 1994.

[2] P. Kremer, T. Wimböck, J. Artigas, S. Schätzle, K. Jöhl, F. Schmidt, C. Preusche, and G. Hirzinger. Multimodal telepresent control of DLR's rollin' JUSTIN. In ICRA'09: Proceedings of the 2009 IEEE international conference on Robotics and Automation, pages 30963097, Piscataway, NJ, USA, 2009. IEEE Press.

[3] K. Minamizawa, S. Fukamachi, H. Kajimoto, N. Kawakami, and S. Tachi. Gravity grabber: wearable haptic display to present virtual mass sensation. In ACM SIGGRAPH 2007 emerging technologies, page 8. ACM, 2007.

[4] K. Minamizawa, D. Prattichizzo, and S. Tachi. Simplified design of haptic display by extending one-point kinesthetic feedback to multipoint tactile feedback. IEEE Haptic Symposium, 2010.

[5] M. Botvinick and J. Cohen. Rubber hands' feel'touch that eyes see. Nature, 391(6669):756-756, 1998.

[6] V.I. Petkova and H.H. Ehrsson. If I were you: perceptual illusion of body swapping. PLoS One, 3(12), 2008. 